Running head: CULTURAL CONSENSUS THEORY

Cultural Consensus Theory as a Method for Evaluating Culturally Shared Beliefs

Natalia Van Doren, M.S. ${ }^{1}$

1370 Moore Building, Department of Psychology, The Pennsylvania State University, University Park, PA 16802 U.S.A; E-mail: nataliavandoren@psu.edu

Note that the current manuscript has not yet been peer-reviewed. 


\begin{abstract}
A central endeavor in the study of culture and psychology is to understand how substantively meaningful cultural variables impact human behavior (e.g., cultural belief systems). Yet, the majority of studies within the field of cultural psychology neglect to do so, instead focusing primarily on group comparisons. One reason for this over-reliance on group comparisons may be the paucity of methods with which to adequately measure cultural variables. In the present paper, I describe a method for evaluating culturally shared phenomena, such as beliefs, based on Cultural Consensus Theory (CCT), and consider the utility of this approach as applied to the study of culture and psychology. Examples as to how this methodology can and has been applied are provided, as are comparisons it to other methods. In doing so, I demonstrate the potential for CCT methods to advance our understanding of cultural beliefs, while acknowledging the limitations inherent in the paradigm.
\end{abstract}




\section{Cultural Consensus Theory as a Method for Evaluating Culturally Shared Beliefs}

Despite early acknowledgement within the field of psychology regarding the influence of culture on human behavior (Wundt, 1921), psychological scientists have struggled to adequately measure this concept. Consequently, researchers frequently use oversimplified metrics to assess culture (e.g., demographic variables), leading to a strong emphasis on group comparisons. As a result, our understanding of substantively meaningful cultural variables (e.g., beliefs; values) remains limited. In the present paper, I will discuss the utility and applications of Cultural Consensus Theory (CCT) as a method for capturing cultural phenomena, such as culturally shared beliefs. Specifically, I argue that CCT provides several advantages over existing methods in that it enables researchers to assess the extent to which cultural beliefs are shared while accounting for within-culture heterogeneity.

\section{What is Culture?}

To start, it is helpful to consider what is meant by "culture". While researchers commonly use sociodemographic variables as proxies for culture (e.g., race; national origin), Betancourt and López (1993) argue that sociodemographic variables do not sufficiently measure culture. Instead, they suggest that culture can be defined as socially shared beliefs, norms, and values within a group, community, or society. This definition also tracks with cognitive theories of culture that define culture as a set of shared meaning systems, or cognitive schemata governing social behavior (D’Andrade, 1995; 1984). There is wide agreement that culture is learned (Triandis, 2007); thus, I argue that shared cultural knowledge is central to the very definition of "culture" itself. 
Despite the centrality of shared knowledge, beliefs, and meaning systems as a key component of culture, the field of cultural psychology remains limited in terms of how to measure culturally shared beliefs, largely because existing methods tend to focus on the individual. This issue is of no trivial importance. Rather, it is central to the study of cultural psychology, which necessitates the understanding that individuals live within a cultural context, and therefore, any study of cultural psychology is inherently hierarchical in nature. In other words, people are nested within cultures, and while it is widely acknowledged that there are mutual paths of influence between individuals and culture, I argue that there is a need within the field of cultural psychology for innovative methods that capture the broader, shared domains of cultural knowledge, whereas present methods focus almost exclusively on examining cultural phenomena at the individual level.

While individual responses can be aggregated to the group level, the predominating methods for response aggregation—namely, simple aggregation (e.g., measures of central tendency), reliability analysis, factor analysis, and even more nuanced methods, like Item Response Theory_-pose several challenges and limitations to the study of culturally shared domain knowledge or beliefs. First, they are item-level methods of aggregation, rather than person-level methods, and therefore focus on measuring an underlying construct that is hypothesized to reside at the individual level as opposed to a shared cultural (i.e., group) level construct. Second, they do not adequately capture individual differences in cultural competence, but instead treat all responses as un-biased and equal. Third, they represent an etic approach to cultural research (e.g., Berry, 1969) by emphasizing cultural comparisons, rather than enabling an organic understanding of cultural phenomena. Thus, in order to move the field forward, I argue for the need to shift from focus on items to persons; from methods that erase within-culture 
heterogeneity to methods that adequately model and capture such heterogeneity; and to methods that take an emic approach to measuring cultural beliefs, all of which are made possible by Cultural Consensus Theory methodology.

\section{What is Cultural Consensus Theory?}

Cultural Consensus Theory (CCT) refers to a set of statistical models for information pooling that were first developed in the 1980s in anthropological and ethnographic research (Romney, Weller, \& Batchelder, 1986; Weller, 2007). CCT provides a framework for measuring culturally shared knowledge and beliefs - that is, the extent to which knowledge or beliefs in a given domain are shared by a group of individuals. Importantly, in this method, "culture" can be defined broadly as any set of individuals who share common knowledge or a common set of beliefs. Thus, while CCT may be applied to more traditional cross-cultural research approaches (e.g., people in China vs. the US) it may also be applied to more localized cultural groups (e.g., gay men in the US; Democrats; sports fans).

Cultural consensus models estimate the culturally appropriate answers to a set of related questions when the answers are unknown, thus requiring no input from the researcher as to the answer key (unlike Item Response Theory, for example), and can simultaneously estimate the individuals' cultural competence, or the degree to which individuals "know" the culturally correct answers (i.e., the consensus) while accounting for item-level characteristics (e.g., item difficulty) and individual differences in cognitive response processes (e.g., guessing tendency). The ability to estimate person-specific levels of cultural competence within CCT is important because aids in estimating the degree to which a set of responses are shared when responses are heterogenous within a group. Weighting is then applied in model estimation such that responses from individuals that are more "culturally competent" are weighted more heavily, thereby 
enabling a reasonable estimate of consensus even with smaller sample sizes, which is particularly helpful when studying hard-to-reach or hard-to-recruit participants (Weller, 2007). Thus, several characteristics of the CCT method make it superior to other aggregation techniques (e.g., measures of central tendency) that would not account for these individual differences.

While a full exposition of the mathematical model(s) used in CCT is beyond the scope of the present paper, I will briefly describe the modeling approach in the interest of explaining both the utility of the method and its applications to cultural psychology. The overall aims of applying a CCT model are to 1) identify whether there are one or more latent cultural groups that share a consensus on answers to a set of questions, 2) assess whether the data supports the model (i.e., assess model fit), and 3) if the model fits well, estimate the model parameters (Romney, Weller, \& Batchelder, 1986). Model parameters include the cultural salience or difficulty of each question, the cultural competence of each participant, and the response biases of each participant (Batchelder, Anders, \& Oravecz, 2018). The output of CCT analyses includes the results for the test of the one-culture assumption (answering the question "Is there a cultural consensus in this domain?"), the answer key (consensus answers to the items; addressing the question "What is the consensus?"), and the aforementioned model parameters (e.g., answering the question "To what extent does each person agree with the group's cultural beliefs?” for cultural competency). Informal models of CCT involve a transposed factor analytic method, such that the one-culture assumption is tested via the person-by-person matrix rather than the item-by-item matrix (Weller, 2007). Formal models in CCT derive from both signal detection theory and Item Response Theory (e.g., Rasch models; Oravecz, Vandekerckhove, \& Batchelder, 2014) and the algorithms used to calculate the consensus and model parameters depend on the type of data that has been gathered (e.g., binary T/F questions vs. Likert responses; Batchelder, Strashny, \& 
Romney, 2010). Many more complex extensions exist, including methods for examining cultural fit ("cultural consonance"; Dressler et al., 2005) and methods for linking cultural competence scores to intensive longitudinal data at the individual level (Oravecz \& Vandekerckhove, 2020).

The main commonality between these related approaches are the research questions to which they can be applied. Importantly, since CCT is a method for estimating shared cultural beliefs, it applies to questions that are applicable to shared, rather than individual, knowledge. For example, the item "Americans think that women are more emotional than men" would be appropriate for CCT analysis; while the item "I think that women are more emotional than men" would not be appropriate, because it measures an individual's endorsement of the item, rather than the individual's understanding of whether a broader cultural group that they belong to would endorse the item.

\section{Comparison with Other Data Aggregation Methods}

To assess the utility of CCT, it is helpful compare and contrast it with other approaches that are used for aggregating responses to a set of questions and for estimating agreement.

Measures of Central Tendency. The simplest and probably most often used technique for measuring cultural beliefs involves administering a survey instrument to a set of individuals and then aggregating the scores to create a mean, group-level score (e.g., Portrait Values Questionnaire; Schwartz et al., 2012). While such measures are intuitive, they fail to adjust for within-culture heterogeneity and can therefore lead to biased estimates. These problems are overcome in CCT because of the use of cultural competence scores and weighting.

Reliability Analysis. Reliability analysis is another standard method for aggregating data and assessing agreement among individuals. A key difference between traditional reliability 
analysis and CCT is the way the estimates of agreement are derived. In reliability analysis, the "answers" to the items (i.e., the means) are estimated first; then, the participant's correspondence to those estimated answers is assessed. In CCT, the consensus answers and cultural competence (individuals' correspondence to the estimated answers) are estimated simultaneously, while also accounting for individual differences in decision-making characteristics (e.g., willingness to guess) and item characteristics (e.g., item difficulty). Because of the simultaneous estimation in the CCT approach, it enables more accurate estimates of the cultural knowledge domain under study compared to traditional reliability analysis.

Factor Analysis. Factor analysis is used in CCT, but in a different way than traditional factor analysis. Specifically, factor-analytic methods (e.g, EFA; PCA) are used as part of the CCT model estimation on the person-by-person correlation matrix to test whether there is a single consensus. If the data bears out this assumption, then the factor analysis should yield a one-factor solution. By contrast, traditional factor analysis would be applied to the item-by-item correlation matrix to test whether there is a single latent trait at the individual level that is best represented by the items. Accordingly, I would argue that when a researcher is interested in understanding cultural beliefs, the CCT approach would be preferable, because it utilizes factor analysis to test of the extent to which such beliefs are shared in a group of people rather than to test the extent to which the individual items assess a latent trait in the individual.

Item Response Theory. CCT and IRT are both psychometric models, but they hold several key differences that, in my view, make CCT the superior method for evaluating culturally shared beliefs. First, CCT is a cognitive psychometric model, meaning that it accounts for cognitive differences in response tendencies, such as guessing bias (e.g., "acquiescence bias") and willingness to guess, and estimates these parameters as person-specific cognitive 
characteristics. While some IRT models can include guessing parameters, the parameter is typically considered as an item characteristic, rather than a person characteristic. Second, CCT models allow for the inclusion of person-level covariates (e.g., sex; personality traits) as predictors of these cognitive response tendencies. Third, IRT models assume that the researcher has prior knowledge of the "correct answers" (i.e., has the "answer key") to the items, while CCT makes no assumptions about the answer key, instead deriving the answer key from the consensus. Additionally, when applied to multicultural data, IRT tests differential item functioning (DIF) by comparing scores across groups, while CCT allows each group to be estimated separately, rather than imposing a structure from one group to another as in DIF. In other words, multicultural CCT models allow each cultural group to have a different answer key (Anders \& Batchelder, 2012), rather than simply measuring the extent to which one group (e.g., a White group) is the same as another cultural group (e.g., a Black group). This is a major advantage if one wishes to take an emic approach to the study of cultural phenomena.

\section{Areas of Application}

Despite its long-standing use in anthropological research (e.g., starting with Batchelder \& Romney, 1988), CCT has only more recently been applied in psychological research. Here, I review briefly several applications of CCT to illustrate the varied ways in which it can be applied to the field of cultural psychology.

Stigma. CCT has been applied to study beliefs about disease causality, which has been associated with the degree to which diseases are stigmatized. For example, Henderson \& Dressler (2017) examined the cultural consensus on causes of addiction. Results revealed that there were two main domain beliefs about addiction causes: a biopsychosocial model, and a moral model (using a model that allows for multiple-consensus truths; Anders \& Batchelder, 
2012). The researchers were then able to use the respondents scores to examine the extent to which addiction causality beliefs link to levels of stigma endorsed towards those with addiction. Participants who endorsed the biopsychosocial model had lower stigma attributions. I chose this example because it illustrates how a similar approach may be applied to examining negative beliefs or stereotypes about outgroups and resulting stigma towards outgroups, a popular topic in the field of cultural and social psychology.

Social Justice. What constitutes "social justice"? While institutions often share common catchphrases that supposedly signal efforts towards equity, the concepts can be quite vague. In a group of college students, CCT was applied to assess what culturally shared understandings students hold regarding the term "social justice", and how this differs in two cultural groups: republicans and democrats (Guest, Lies, Kerssen-Griep, \& Frieberg, 2009). The researchers found that while the consensus was largely similar around issues of diversity, equity, and inclusion, democrats also included environmental concerns in their definition of social justice, while republicans did not. The implications of such findings illustrate the utility of a CCT approach to the study of multicultural data. In this case, understanding more about the consensus on social justice in different student groups could aid university officials in understanding how to best meet the needs of a diverse student body. Similarly, understanding the cultural consensus on shared definitions of concepts could aid in cultural psychological research focused on how to best address the needs of a diverse group of individuals in other domains (e.g., graduate school training; mental health treatment).

Racism. Of particular interest, a recent study (Michaels, Gallagher, Crawford, Kanter, \& Williams, 2018) applied CCT to examine consensus in terms of what behaviors are considered racial microagressions in three groups of participants: diversity experts, Black individuals, and 
White individuals. Participants were presented with cross-racial interaction scenarios (vignettes) followed by a set of items capturing possible behavioral responses. Rating scales differed by participant groups: while the diversity experts and Black individuals were asked to rate the extent to which each response was microagressive on a Likert scale from very racist to not racist / supportive, White participants were asked to rate the likelihood that they would engage in the behavior. The data were analyzed using CCT (implemented with a Latent Truth Rater Model) to examine the following: 1) the degree to which the expert panel demonstrated a consensus on rating the responses as racist vs. supportive; 2) the degree of consensus amongst Black participants in rating responses as racist vs. supportive; 3) the degree of consensus amongst White participants in their endorsement of how likely it is that they would engage in the reported behaviors; and finally, 4) the between-group correlations in terms of their consensus answers.

This example nicely illustrates several ways in which CCT enabled the testing of research questions regarding cultural beliefs that could not have been estimated as well using another method. First, CCT enabled the researchers to test three different cultural groups to estimate their agreement separately, as well as the correlations between their answer keys. Second, the rating scales for each group were allowed to differ — while experts and Black individuals rated items as racist vs. supportive, White individuals instead were asked to rate the degree to which they would engage in the behavior, thereby enabling the researchers to gain a complex and nuanced understanding of the cultural construct of "microaggressions" by examining appraisals from potential perpetrators (White individuals), victims (Black individuals), and experts.

\section{Limitations}

There are several limitations that warrant mention. While CCT is generally more conducive to an emic approach, researchers may still insert bias in terms of the item selection. In 
other words, researchers still select the items that are used for the consensus analysis, which could introduce imposed etics and incomplete measurement of the domain. Thus, in order to be applied optimally for bottom-up approaches to cultural research, I recommend researchers combine the method with other approaches, such as pilot focus groups, qualitative interview methods, or consultation with expert panels to be maximally effective.

The second is that cultural groups do have to be defined a priori (e.g., sex; race). However, unlike other methods of aggregation, the model actually tests whether individuals within a group do reflect a shared culture. In other aggregation methods, that is assumed. Furthermore, CCT would also show if there is a subculture (i.e., a two-culture solution) within a group selected a priori, which could then be subjected to further analysis, whereas in other approaches, the group would be treated as a monolith.

\section{Conclusion}

Although CCT has been applied to ethnographic research since the 1980s, the application of CCT to the field of psychology has been more recent and is somewhat limited, perhaps due to the preponderance of individual-level approaches to the study of cultural psychology. I argue that the utility and relevance of this approach warrants further application to many areas of cultural psychology that are typically studied using methods that are psychometrically similar, but not sufficient to assess the cultural level of analysis. In sum, CCT has the potential to advance the field of cultural psychology by enabling a more accurate assessment of cultural constructs. Such an approach can help the field to shift towards a focus more in line with the purported goal of cultural psychology, which is to study the individual in a cultural context and how the individual is affected by culture (and vice versa) - namely, by formalizing the measure of culture itself, 
through examining culturally shared beliefs in relevant domains of interest. Put simply, it is time we brought "culture" back into the study of cultural psychology. 


\section{References}

Anders, R., \& Batchelder, W.H. (2012). Cultural consensus theory for multiple consensus truths. Journal of Mathematical Psychology, 56(6), 452-469. doi:10.1016/j.jmp.2013.01.004

Batchelder, W. H., \& Romney, A. K. (1988). Test theory without an answer key. Psychometrika, 53(1), 71-92. doi:10.1007/bf02294195

Batchelder, W. H., Strashny, A., \& Romney, A. K. (2010). Cultural Consensus Theory: Aggregating Continuous Responses in a Finite Interval. Advances in Social Computing Lecture Notes in Computer Science, 98-107. doi:10.1007/978-3-642-12079-4_15

Batchelder, W. H., Anders, R., \& Oravecz, Z. (2018). Cultural consensus theory. In J. Wixted, \& E. J. Wagenmakers (Eds.), The Stevens' handbook of experimental psychology and cognitive Neuroscience, Vol. V: Methodology (pp. 221-264). New York, NY: John Wiley \& Sons.

Berry, J. W. (1969). On cross-cultural comparability. International Journal of Psychology, 4(2), 119-128. doi:10.1080/00207596908247261

Betancourt, H., \& López, S. R. (1993). The study of culture, ethnicity, and race in American psychology. American Psychologist, 48(6), 629-637. doi:10.1037/0003-066x.48.6.629

D’Andrade, R. (1995). The Development of Cognitive Anthropology. Cambridge: Cambridge University Press.

D’Andrade, R. (1984). Cultural Meaning Systems. In Culture Theory: Essays on Mind, Self, and Emotion. Cambridge: Cambridge University Press.

Dressler, W. W., Borges, C. D., Balieiro, M. C., \& Santos, J. E. (2005). Measuring Cultural Consonance: Examples with Special Reference to Measurement Theory in Anthropology. Field Methods, 17(4), 331-355. doi:10.1177/1525822x05279899 
Guest, A. M., Lies, J. M., Kerssen-Griep, J., \& Frieberg, T. J. (2009). Concepts of Social Justice as a Cultural Consensus: Starting Points for College Students of Different Political Persuasions. Journal of College and Character, 10(6). doi:10.2202/1940-1639.1446

Henderson, N. L., \& Dressler, W. W. (2017). Medical Disease or Moral Defect? Stigma Attribution and Cultural Models of Addiction Causality in a University Population. Culture, Medicine, and Psychiatry, 41(4), 480-498. doi:10.1007/s11013-017-9531-1

Michaels, T. I., Gallagher, N., Crawford, M., Kanter, J. W., \& Williams, M. T. (2018). Racial differences in the appraisal of microaggressions through cultural consensus modeling. The Behavior Therapist, 41, 314-321.

Oravecz, Z., \& Vandekerckhove, J. (2020). A joint process model of consensus and longitudinal dynamics. Journal of Mathematical Psychology, 98, 102386. doi:10.1016/j.jmp.2020.102386

Oravecz, Z., Vandekerckhove, J., \& Batchelder, W. H. (2014). Bayesian Cultural Consensus Theory. Field Methods, 26(3), 207-222. doi:10.1177/1525822x13520280

Romney, A. K., Weller, S. C., \& Batchelder, W. H. (1986). Culture as Consensus: A Theory of Culture and Informant Accuracy. American Anthropologist, 88(2), 313-338. doi:10.1525/aa.1986.88.2.02a00020

Triandis, H. C. (2007). Culture and psychology: A history of the study of their relationship. In S. Kitayama \& D. Cohen (Eds.), Handbook of cultural psychology (p. 59-76). The Guilford Press.

Weller, S. C. (2007). Cultural Consensus Theory: Applications and frequently asked questions. Field Methods, 19(4), 339-368. doi:10.1177/1525822x07303502

Wundt, W. (1921). Völkerpsychologie: Vols. 1-10. Leipzig, Germany: Alfred Kroner Verlag. 